\title{
Load capacity of the mixed bench and slab foundation. Numerical simulations and analytical calculation model
}

https://doi.org/10.2478/sgem-2021-0005

received May 20, 2020; accepted March 3, 2021.

\begin{abstract}
The paper presents results of a numerical investigation on load capacity of the mixed bench and slab shallow foundations (often used in the process of the modernization of the old, antique buildings, which are suffering from lack of the load capacity). The main trouble with use of existing analytical approaches is a non-unique foundation level of the bench and slab, they could even be founded on different geotechnical layers. Proposed analytical model based on Brinch Hansen (EC-7) approach could deal with such a problem. Results of 2D and 3D numerical modelling (ultimate load of the foundation) are compared to the obtained by using the proposed approach. Influence of the soil above the foundation level is also investigated. Different width to length ratios of the foundation are analyzed (from "short" to "long" foundations). Usability of the proposed analytical model in engineering practice is proved by numerical simulations; the obtained results are on the safe side with quite acceptable margin of additional safety.
\end{abstract}

Keywords: Bench and slab foundation; load capacity; numerical modelling.

\section{Introduction}

\subsection{Definition of the problem}

Old, antique buildings are often founded on stone, masonry or concrete benches (Fig. 1). Modernization of the structure (to meet today's standards) often leads to situation where load capacity of the existing foundation benches is inadequate in comparison with loading. This is

* Corresponding author: Michat Grodecki, Civil Engineering Department, Cracow University of Technology, Warszawska 24, 31155 Cracow, Poland, E-mail: mgrode@pk.edu.pl

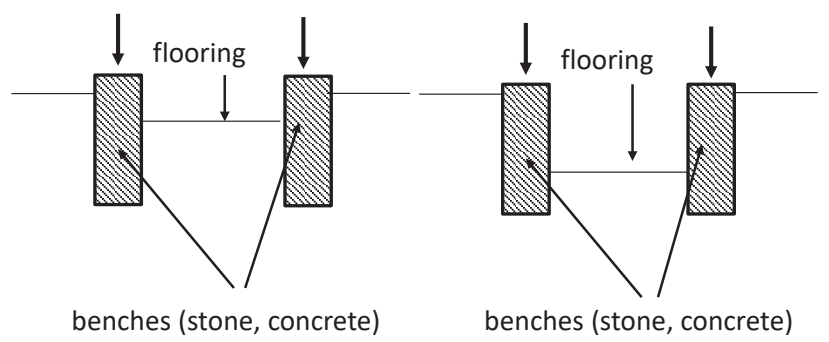

Figure 1: Typical foundation of the antique building (without or with basement).

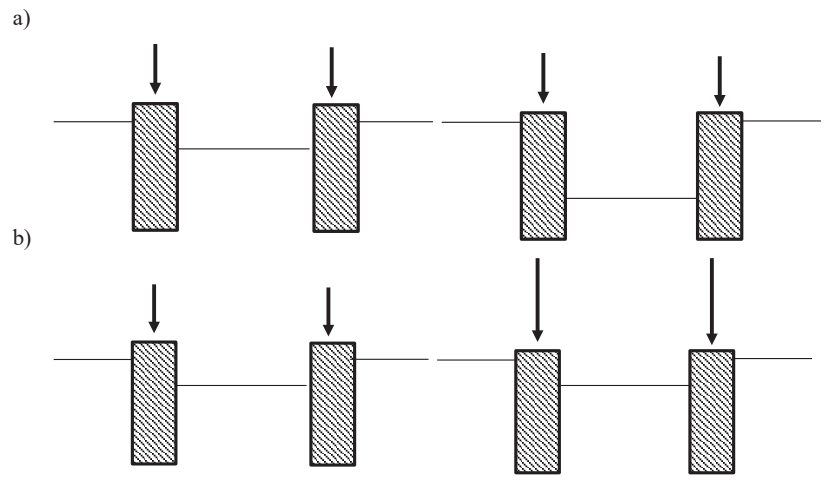

Figure 2: Sources of the foundation load capacity deficiency: a) basement deepening, b) load increase.

mainly due to load increase (when for example additional story is designed or way of the usage of the building is changed) or load capacity decreased (when basement is deepened). Sometimes these two situations occur simultaneously (Fig. 2).

In order to deal with such a problem, to increase load capacity of the existing foundation benches, several possible remedies are used (Fig. 3).

Dimensions of the existing foundations' benches could be increased (both in horizontal and vertical direction). Butsuch a technology requires digging out of the foundations, which is dangerous not only to the building structure itself, but also to the buildings in the vicinity. 
a)

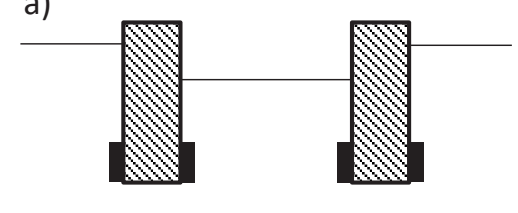

c)

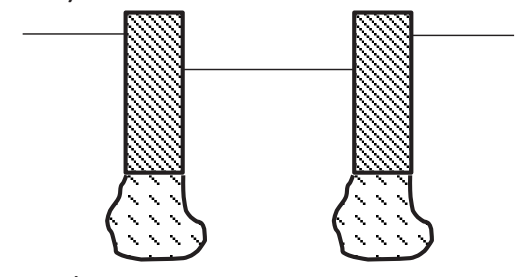

e)

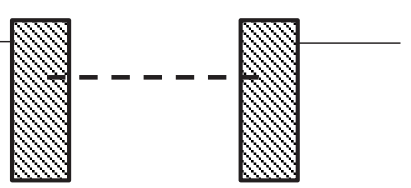

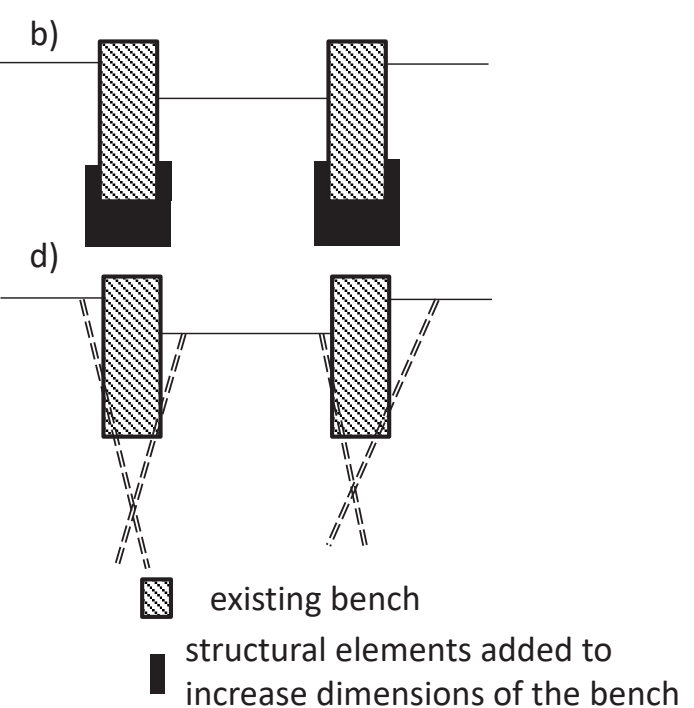

S pressure injection $====$ micropiles

- - additional foundation slab

Figure 3: Remedies used to increase the load capacity of the existing foundation: increase of the existing foundation dimension: a) horizontal, b) horizontal and vertical, c) pressure injection, d) micropiles installation, e) addition of the reinforced concrete foundation slab.

Limited available space (especially in urban environment) is also a serious problem in this technology. Another remedy is usage of the pressure injection or micropiles under the existing foundation benches. This method could be performed only by specialized geotechnical companies, which have a lot of experience in such a work. And still some danger for building structure itself and for buildings in the vicinity exists. Fourth possible method is to design additional reinforced concrete foundation slab between the existing foundation benches. Such a slab must be connected with existing benches, in order to form a united foundation. This is the easiest and cheapest method to increase the load capacity of the foundation. It does not require any additional excavations. But some design problems appear:

1. How to calculate the ultimate load of such a foundation when benches and slabs are on different levels, especially when soil conditions on the foundation level of the benches and slab are different?

2. How to calculate the internal forces (bending moments and shear forces) in the slab?

The second problem could be solved using the wellknown slab on Winkler subsoil model. The first one is a main subject of this article.

\subsection{Existing analytical calculations models for direct foundations' ultimate load calculations}

Problem of calculation of the shallow foundation ultimate load is very old. First works by Prandl (1920, 1921), later adopted by Reissner (1924), Keverling Buisman (1940) and Terzaghi (1943) lead to a simple analytical equation for the calculation of ultimate load. This approach, a bit modified by Meyerhof $(1951,1953,1963)$ and Brinch Hansen (1970) is adopted by many codes, for example, by Eurocode-7 (EC-7). Outline of this approach (deterministic one) is presented below:

$$
\begin{gathered}
q=c N_{C} b_{C} s_{C} i_{C}+q^{\prime} N_{q} b_{q} s_{q} i_{q}+0.5 \gamma B N_{\gamma} b_{\gamma} s_{\gamma} i_{\gamma} \\
N_{q}=e^{\pi \operatorname{tg} \varphi} \operatorname{tg}^{2}(45+\varphi / 2) \\
N_{c}=\left(N_{q}-1\right) \operatorname{ctg} \varphi \\
N_{\gamma}=2\left(N_{q}-1\right) \operatorname{tg} \varphi
\end{gathered}
$$

where: $\mathrm{q}$ - ultimate load of the foundation $[\mathrm{kPa}], c-$ cohesion of the subsoil [kPa], $N_{q}, N_{c}, N_{g}$ - bearing capacity coefficients [-], $b_{c}, b_{q}, b_{\gamma}$ - coefficients of load inclination, 
for vertical load $=1[-], s_{c}, s_{q}, s_{\gamma}$ - coefficients of the foundation shape, for bench $=1[-]$

$$
\begin{gathered}
s_{q}=1+B / L \sin (\varphi) \\
s_{\gamma}=1-0.3 B / L \\
s_{c}=\left(s_{q} N_{q}-1\right) /\left(N_{q}-1\right)
\end{gathered}
$$

$i_{c}, i_{q}, i_{\gamma}-$ coefficients of the foundation inclination, for horizontal foundation $=1 \quad[-]$, $q^{-}$- vertical stress on the foundation level outside of the foundation $[\mathrm{kPa}], \phi$ - internal friction angle of the subsoil $\left[{ }^{\circ}\right], \mathrm{B}$ - foundation width [m], $\mathrm{L}$ - foundation length [m], $\gamma$ - soil volumetric weight $\left[\mathrm{kN} / \mathrm{m}^{3}\right]$.

It's worth noting that the equations for calculating the bearing capacity coefficients (as a functions of the internal friction angle) are still a subject of the investigations (Edgar Giovanny (2013), van Bars (2014)). Uncertainties of this model were discussed by Motra et al. (2016).

Wide discussion on the problem of partial material and load factors used in engineering calculations of direct foundations' ultimate load is given by Bogusz and Godlewski (2019).

In the last few years, special attention was paid to the spatial variability of soil properties (mainly strength parameters-internal friction angle and cohesion), which leads to the usage of probabilistic approach in foundation safety investigation. In the work of Puła and Chwała (2015), soil strength parameters are considered as random fields, later averaged alongside the Prandtl's slip line. Random character of the slip line (as an effect of the random friction angle) is taken into account. Similar approach (but used to deal with seismic bearing capacity of strip footing) is presented by Johari et al. (2017). In the work of Johari et al. (2019), Young's modulus is treated as a random field in the analysis of differential settlement of strip footing. Stochastic finite element method (SFEM) is used. But linear elastic model is used to represent the soil behavior. This is far from the real soil behavior (nonlinearly-elastic in the small strain range and plastic close to the ultimate state).

But in the aforementioned methods for foundation's ultimate load estimation, a constant level of foundation (strictly speaking-constant vertical compressive stress on the foundation level outside of the foundation) is assumed, which is very often not true in the case of mixed bench and slab foundations. As far as the Author knows, there is no analytical solution for this problem in the literature.

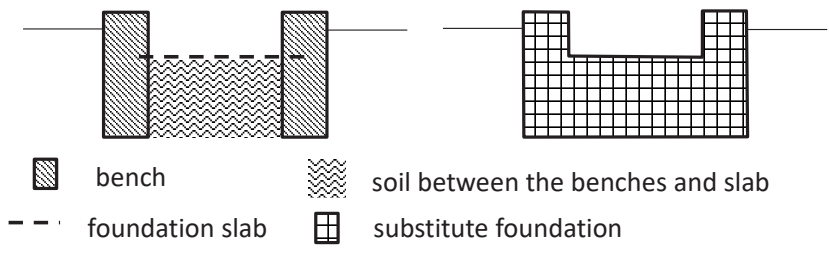

Figure 4: Proposed substitute foundation for benches, slab and soil between them.

\section{Proposed calculation method}

Proposed method for bench and slab foundation ultimate load estimation is based on Brinch Hansen approach, adopted in EC-7. First, a very conservative idea, was to calculate ultimate load only for slab, neglecting the effect of the benches. This is acceptable from an engineering point of view, because it leads to safe results (to underestimate the ultimate load of the structure). But in some cases (when benches are founded much deeper than slab, on high capacity soil, and slab is founded on weak soil), this approach leads to obtain uneconomical results. Sometimes the obtained ultimate load of the slab is lower than for benches alone! So another, more sophisticated approach is proposed. Soil under the slab and between the benches could be treated as a rigid body. So, the mixture of slab, benches and soil forms the substitute foundation, with a constant foundation level (Fig. 4). Ultimate load of such a foundation could be easily calculated with the use of Brinch Hansen (or any similar) approach. Weight of the soil between the benches and the slab should be added to the foundation loading. In order to verify if such a proposition leads to obtain reasonable results, numerical verification is performed.

\section{Numerical verification}

In order to verify the above-proposed calculation method, numerical simulations were performed. FEM system ZSoil v18 was used. Elastic-plastic Mohr-Coulomb with cut-off condition (no tension) model was used to model the soil and elastic model for foundation. It is of course possible to use a more sophisticated model for soil (for example, Hardening Soil model). But it's not necessary when the main topic of interest is the ultimate load of the structure. Interface elements with no friction between foundation and soil were used to model the discontinued displacement field. For slab, beam elements were used in 2D plane strain analysis and shell elements in 3D analysis. 


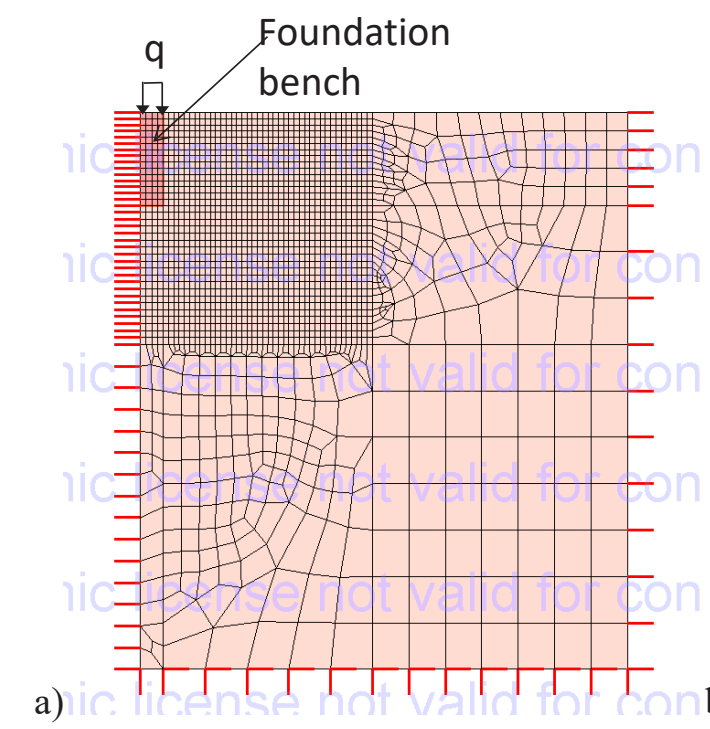

b)

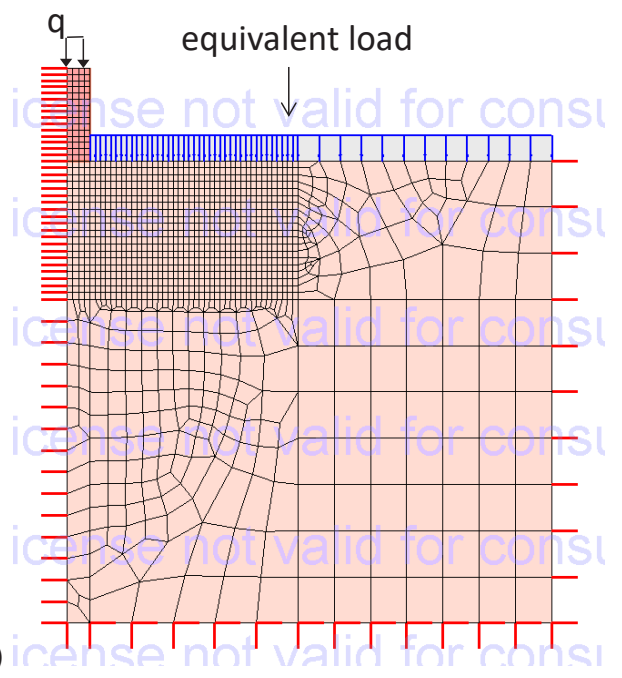

Figure 5: 2D numerical model of the simple single bench with boundary conditions-with soil above foundation level modelled as: a) a CoulombMohr continuum, b) an equivalent load.

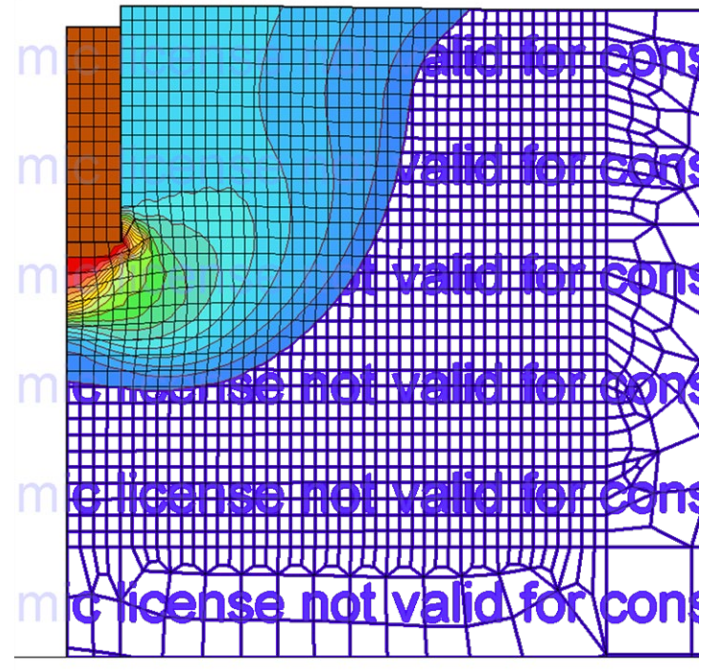

olowkonv_02 Date: 19.2 .2018 12:0

Figure 6: Failure mode of the single bench.

Problem was run as a force-driven one. External load of the foundation was increased as long as a divergence of the iterative procedure occurs. Settlement - load curves were checked in order to judge if the divergence of the iterative process is caused by reaching of the ultimate load or only by some numerical instability (see Fig. 7).

First of all, the simple single bench ultimate load problem was considered, in order to compare the numerically obtained value of ultimate load with the results of Brinch Hansen (EC-7) approach. Foundation bench with $1 \mathrm{~m}$ width founded at $2 \mathrm{~m}$ below the terrain level on homogenous subsoil (medium capacity clay, see Table 1) was considered. 2D plane strain half-model was used to speed up the calculations (Fig. 5). Model consisted of 1957 Q4 2D-continuum elements, 2065 nodes and 19 interface elements for soil above the foundation level modelled as a continuum and 1271 Q4 2D-continuum elements, 1369 nodes and 4 interface elements for soil above the foundation level modelled as an equivalent load.

Obtained value of ultimate load $280 \mathrm{kPa}$ is significantly higher than the results of EC-7 calculations (187.5 kPa), difference is about $49 \%$. Possible source of such a big difference is treating a soil above the foundation level as a source of the vertical compressive stress in the Brinch Hansen (EC-7) approach. So, the numerical simulation with soil above foundation level replaced by equivalent vertical load was performed. Obtained value of ultimate load $202 \mathrm{kPa}$ is a bit higher than the results of EC-7 calculations (187.5 kPa), difference is about $8 \%$. So, for simple problems, the numerical calculation gives values of an ultimate load quite close to those obtained from the Brinch Hansen (EC-7) approach, if soil above the foundation level is treated as a source of vertical stress only.

In the simulations presented in the later part of this paper, both approaches to the problem of soil above the foundation level (modelling as a Coulomb-Mohr continuum and as an equivalent load) are used, and the obtained results are compared. 


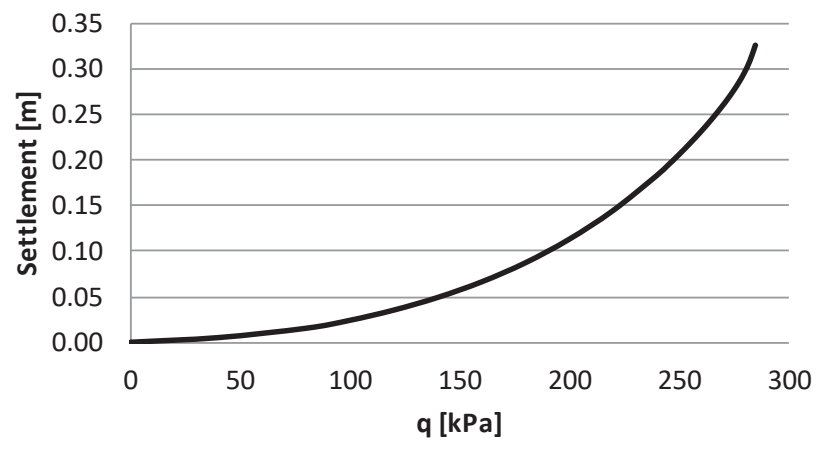

Figure 7: Example of the load-settlement curve, ultimate load reached.

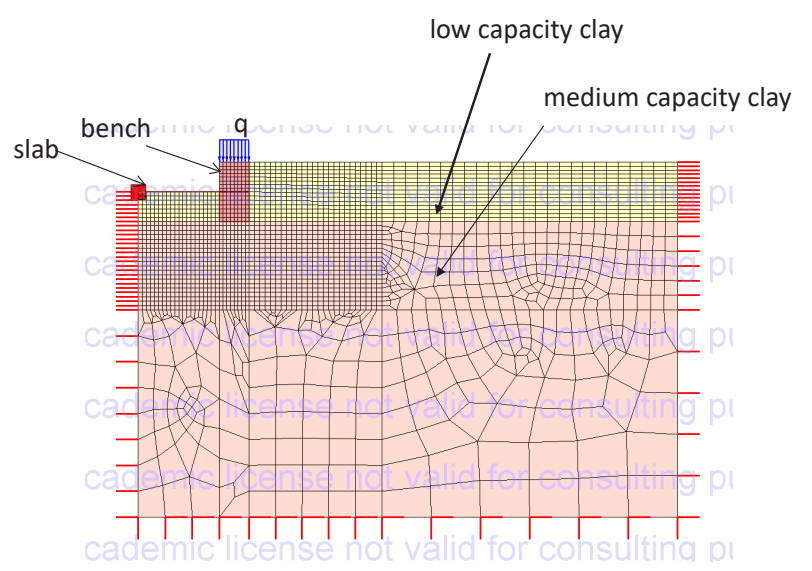

Figure 8: 2D numerical model of the bench and slab with boundary conditions.

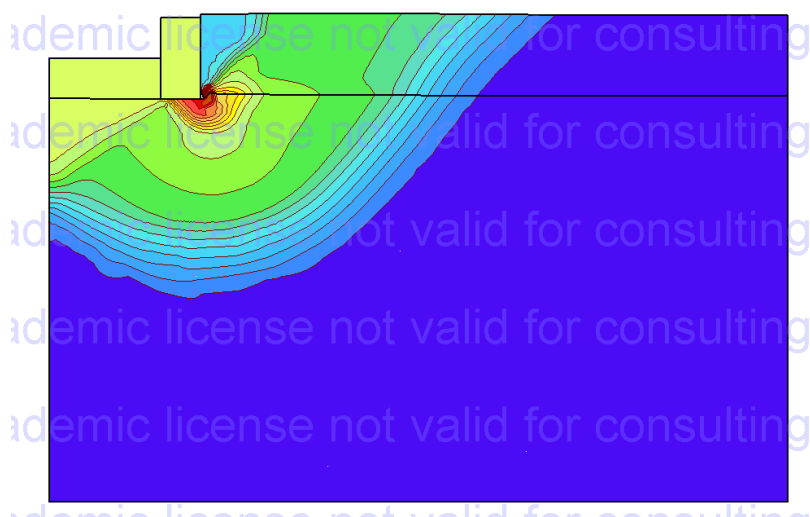

Figure 9: Failure mode of the bench and slab foundation, 2D calculations result.

Obtained failure mode is similar to those considered in the analytical solutions; triangle under the bench is visible in Fig. 6.
Table 1: Parameters of soil used in the bearing capacity analysis.

\begin{tabular}{lllll}
\hline Material & $E$ & $\gamma$ & $\phi$ & $c$ \\
& {$[\mathrm{MPa}]$} & {$\left[\mathrm{kN} / \mathrm{m}^{3}\right]$} & $\begin{array}{l}\left.{ }^{0}\right] \\
{[\mathrm{kPa}]}\end{array}$ \\
\hline Medium capacity clay & 15 & 20 & 10 & 10 \\
Low capacity clay & 5 & 18 & 5 & 5 \\
\hline
\end{tabular}

Then a more complicated case was analyzed. 2D plane strain half model of the benches and slab foundation (which corresponds to long foundation bench, $B / L \rightarrow 0$ ) was built. $1 \mathrm{~m}$ width benches were founded on medium capacity clay $2 \mathrm{~m}$ below the terrain level, where $5.5 \mathrm{~m}$ width $30 \mathrm{~cm}$ thick slab-on low capacity clay $1 \mathrm{~m}$ below the terrain level (typical situation for antique buildings, see Table 1 and Fig. 8).

Model consisted of 2532 Q4 2D-continuum elements, 2694 nodes, 28 beam elements and 50 interface elements for soil above the foundation level modelled as a continuum and 1782 Q4 2D-continuum elements, 1915 nodes 28 beam elements and 35 interface elements for soil above the foundation level modelled as an equivalent load.

Obtained value of ultimate load $263.5 \mathrm{kPa}$ (252 kPa with equivalent load approach) is a bit higher than the results of the proposed calculations method $(221.5 \mathrm{kPa})$, difference is about $19 \%$ (14\% with equivalent load approach). Obtained failure mode is illustrated in Fig. 9. Ultimate load of the slab itself (founded on a weak soil) calculated according to Brinch Hansen (EC-7) is $71.3 \mathrm{kPa}$; so such a conservative approach leads to great underestimation of the ultimate load, which is unacceptable from an economical point of view.

Also, 3D calculations of the $1 / 4$ of the real structure were performed (see numerical model shown in Fig. 10), in order to verify if the proposed calculations method works not only for long $(B / L \rightarrow 0)$ benches but also for situations where coefficients of the foundation shape have a significant influence on the obtained values of ultimate load.

Biggest of the used 3D model consisted of 27280 Q8 3D-continuum elements, 32296 nodes, 440 shell elements and 933 interface elements (soil above the foundation level modelled as a continuum, "long" foundation with B $=7.5 \mathrm{~m}$ and $\mathrm{L}=28 \mathrm{~m}$ ), where the smallest one consisted of 16040 Q8 3D-continuum elements, 19661 nodes, 104 shell elements and 693 interface elements (soil above the foundation level modelled as an equivalent load, "short" foundation with $B=7.5 \mathrm{~m}$ and $\mathrm{L}=7.5 \mathrm{~m}$ ). 


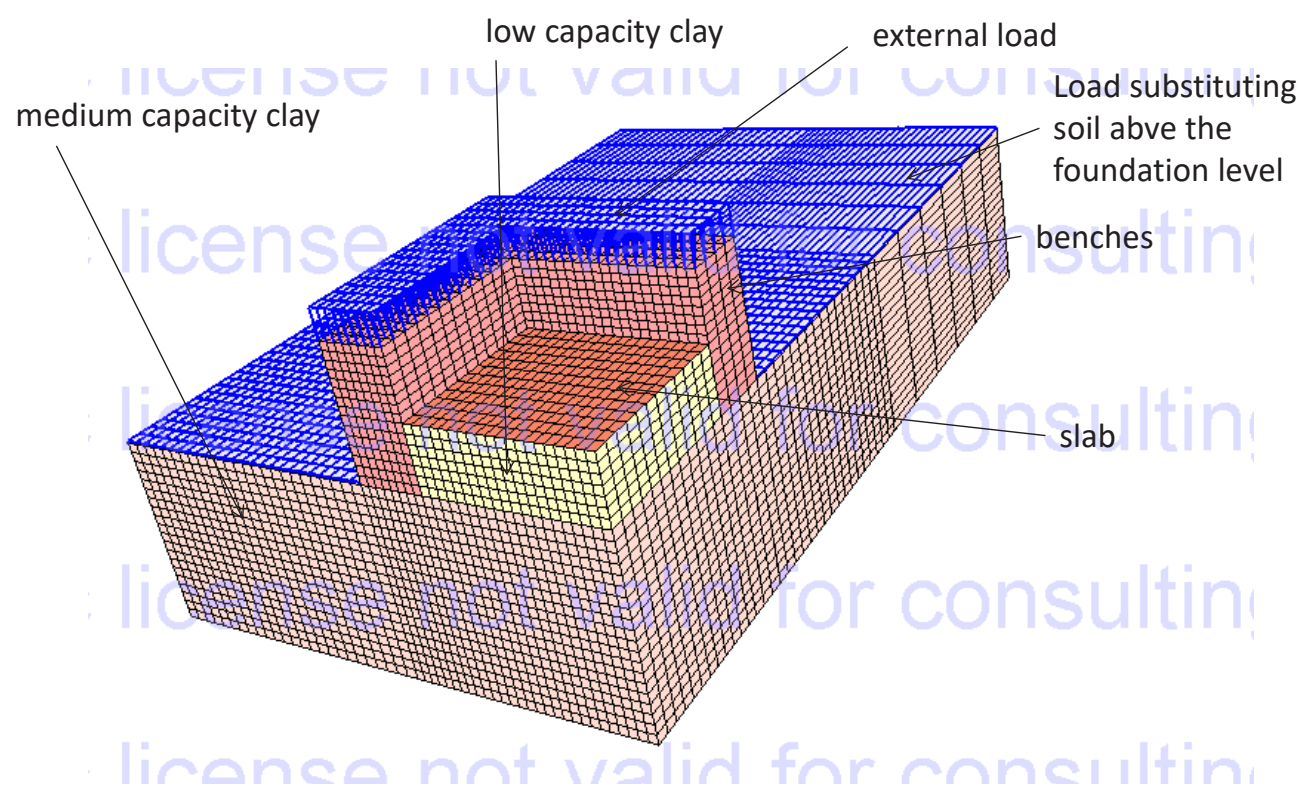

Figure 10: 3D numerical model of the bench and slab, equivalent load approach.

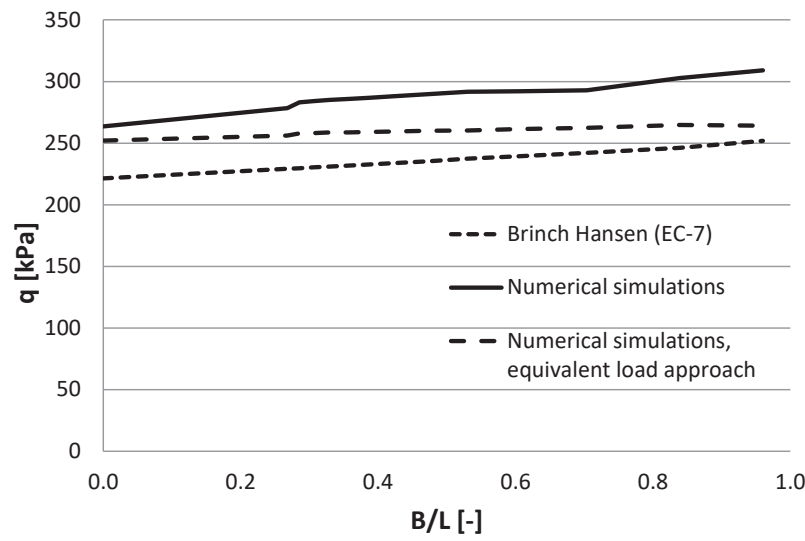

Figure 11: Obtained values of ultimate load as a function of $B / L$ ratio.

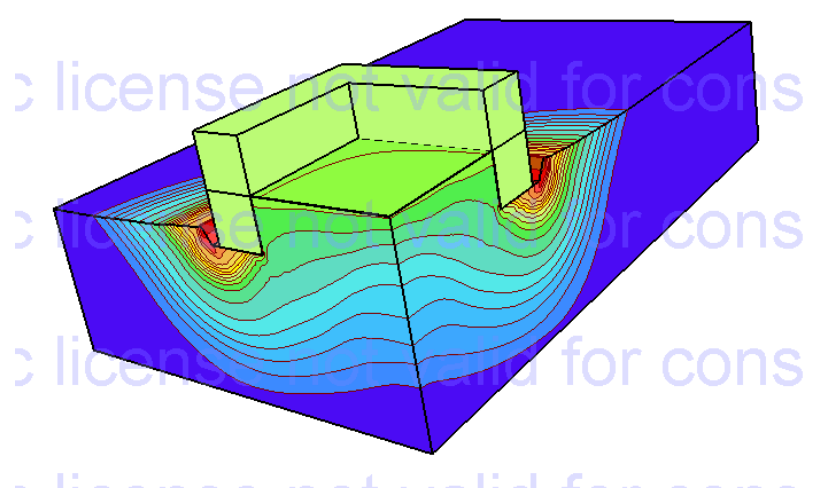

Obtained result are illustrated in Fig. 11.

Obtained results show that treating soil above the foundation level as a Coulomb-Mohr continuum leads to obtain values of ultimate load about $17-19 \%$ higher than those obtained from the proposed approach. It's quite acceptable from an engineering and economical point of view. When using an equivalent load approach, differences drops down to 14\% for "long" foundations and to about $5 \%$ for "short" $(\mathrm{B} / \mathrm{L}=1)$ ones. Obtained failure mode is similar to those observed in 2D simulations (Fig. 12).

Obtained load - settlement curves are almost identical for any points located on the bench. This is due to high stiffness of the bench. Maximal settlement difference is observed between the bench and the central point of the slab. The difference tends to vanish in the ultimate state (see Fig. 13). However, the values of the settlements presented here are only rough approximation of the reality. This is due to the simple constitutive CoulombMohr model used and not taking into account the stiffness of the whole building. More sophisticated model, for example, Hardening Soil model, could be used to model the settlement process closer to the reality, but it will not change the obtained values of ultimate load.

Figure 12: Failure mode of the bench and slab foundation, 3D calculations result. 


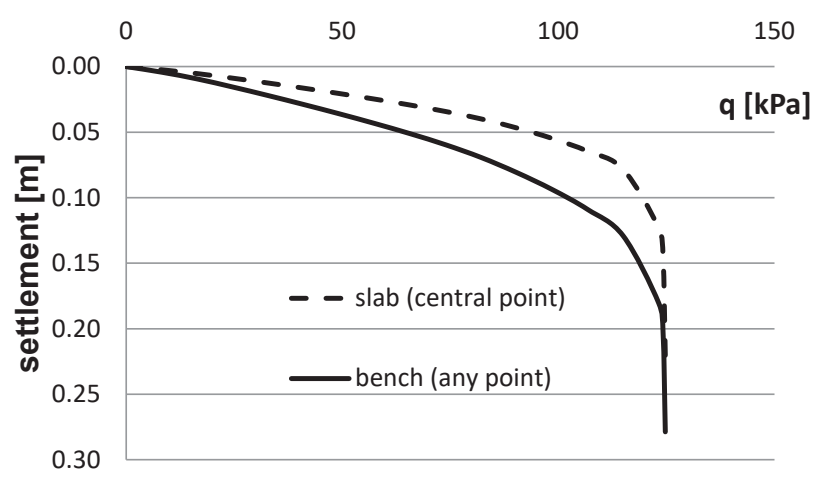

Figure 13: Example of load-settlement curves for bench and central point of the slab-results of the 3D calculations.

\section{Conclusions}

Presented approach allows to adopt the Brinch-Hansen approach to the mixed bench and slab foundations. Calculated values of the ultimate load are a bit lower than those obtained from numerical simulations. Difference is between $17 \%$ and $19 \%$ when soil above the foundation level is treated (in numerical simulations) as a Coulomb-Mohr continuum and drops down to $5 \%$ for "short" $(\mathrm{B} / \mathrm{L}=1)$ foundations if equivalent load approach is used. Proposed approach works both for "short" $(\mathrm{B} / \mathrm{L}=1)$ and long $(\mathrm{B} / \mathrm{L}=$ 0 ) foundations. So it can be used in engineering practice. Influence of the soil above foundation level on ultimate load of the structure is an additional margin of safety.

\section{References}

[1] Bogusz, W., Godlewski (2019), T. Philosophy of geotechnical design in civil engineering - possibilities and risks. Bulletin of the Polish Academy of Sciences Technical Sciences. 67(2), 289-306

[2] Brinch Hansen, J. (1970) A revised extended formula for bearing capacity. Danish Geotechnical Institute Bulletin. 28, 5-11

[3] Edgar Giovanny, D.S. (2013) Assessment of the range of variation of $\mathrm{N} \gamma$ from 60 estimation methods for footings on sand. Canadian Geotechnical Journal. 50, 793-800

[4] Johari, A., Hosseini, S.M., Keshavarz A. (2017) Reliability analysis of seismic bearing capacity of strip footing by stochastic slip lines method. Computers and Geotechnics. 91, 203-217

[5] Johari, A., Sabzi A., Gholaminejad A. (2019) Reliability analysis of differential settlement of strip footings by stochastic response surface method (2019) Iran J Sci Technol Trans Civ Eng. 43,37-48

[6] Keverling Buisman, A.S. (1940) Grondmechanica. Waltman, Delft, the Netherlands
[7] Meyerhof, G.G. (1951) The ultimate bearing capacity of foundations. Géotechnique. 2, 301-332

[8] Meyerhof, G.G. (1953) The bearing capacity of foundations under eccentric and inclined loads. In: Proceedings of the III International Conference on Soil Mechanics and Foundation Engineering, Zürich, Switzerland, 440-445

[9] Meyerhof, G.G. (1963) Some recent research on the bearing capacity of foundations. Canadian Geotechnical Journal. 1(1),16-26.

[10] Motra, H.B., Stutz, H., Wuttke, F. (2016) Quality assessment of soil bearing capacity factor models of shallow foundations. Soils and Foundations. 56(2), 265-276

[11] Prandtl, L. (1920) Ueber die Haerte plastischer Koerper. Nachrichtender Gesellschaftder Wissenschaften. Berichte Mathem. -Phys. Kl, 74-85

[12] Prandtl, L. (1921) Ueberdie Eindringfestigkeit (Haerte) plastischer Baustoffeund die Fes-tigkeit von Schneiden. Z. Angew. Math. Mech.1. Band1,15-20

[13] Puta, W., Chwała M. (2015) On spatial averaging along random slip lines in the reliability computations of shallow strip foundations. Computers and Geotechnics. 68, 128-136.

[14] Reissner, H. (1924) Zum Erddruckproblem. In: Biezeno, C.B.,Burgers, J.M. (Eds.), Proceedings of the 1st International Congress for Applied Mechanics, Delft, The Netherlands: 295-311

[15] Terzaghi, K. (1943) Theoretical Soil Mechanics. J. Wiley, New York.

[16] van Baars, S. (2014) The inclination and shape factors for the bearing capacity of footings. Soils and Foundations. 54(5), 985-992 